TRANSACTIONS OF THE

AMERICAN MATHEMATICAL SOCIETY

Volume 352, Number 2, Pages 731-751

$\mathrm{S}$ 0002-9947(99)02336-3

Article electronically published on October 6, 1999

\title{
REMARKS ON A NONLINEAR PARABOLIC EQUATION
}

\author{
MATANIA BEN-ARTZI, JONATHAN GOODMAN, AND ARNON LEVY
}

\begin{abstract}
The equation $u_{t}=\Delta u+\mu|\nabla u|, \mu \in \mathbb{R}$, is studied in $\mathbb{R}^{n}$ and in the periodic case. It is shown that the equation is well-posed in $L^{1}$ and possesses regularizing properties. For nonnegative initial data and $\mu<0$ the solution decays in $L^{1}\left(\mathbb{R}^{n}\right)$ as $t \rightarrow \infty$. In the periodic case it tends uniformly to a limit. A consistent difference scheme is presented and proved to be stable and convergent.
\end{abstract}

\section{INTRODUCTION}

In this paper we consider the Cauchy problem for the semilinear parabolic equation

$$
\begin{aligned}
& u_{t}=\Delta u+\mu|\nabla u|, \quad 0 \neq \mu \in \mathbb{R}, \\
& u(x, 0)=u_{0}(x),
\end{aligned}
$$

in $\mathbb{R}^{n}$ as well as in the periodic case. Throughout the paper we denote by $\nabla u$ the gradient with respect to the $x$ coordinates.

We treat the questions of existence, uniqueness, regularity and long time decay for initial conditions in $L^{1}$.

In the case of $\mathbb{R}^{n}$ and very smooth initial condition, say $u_{0} \in C_{0}^{4}\left(\mathbb{R}^{n}\right)$ (i.e. four times continuously differentiable with compact support), the global existence, uniqueness and decay properties for solutions of (1.1), have been proved in [B] as follows.

Theorem 1.1. There exists a unique classical solution of (1.1) in $\mathbb{R}^{n} \times[0, \infty)$. This solution satisfies the maximum principle,

$$
\begin{aligned}
& \sup _{\mathbb{R}^{n} \times[0, \infty)} u(x, t)=\sup _{\mathbb{R}^{n}} u_{0}(x), \inf _{\mathbb{R}^{n} \times[0, \infty)} u(x, t)=\inf _{\mathbb{R}^{n}} u_{0}(x), \\
& \sup _{\mathbb{R}^{n} \times[0, \infty)}|\nabla u(x, t)| \leq \sum_{i=1}^{n} \sup _{\mathbb{R}^{n}}\left|\frac{\partial u_{0}}{\partial x_{i}}(x)\right| .
\end{aligned}
$$

Theorem 1.2. Let $u_{0}(x) \geq 0$ and $\mu<0$. Then the solution $u(x, t)$ decays as $t \rightarrow \infty$ in the following sense.

There exists a constant $A_{n}>0$, depending only on $n$ and the support of $u_{0}$, such that

$$
t^{(|\mu| /(1+|\mu|)) A_{n}} \int_{\mathbb{R}^{n}} u(x, t) d x
$$

remains bounded as $t \rightarrow \infty$.

Received by the editors November 11, 1996 and, in revised form, September 22, 1997.

1991 Mathematics Subject Classification. Primary 35K15, 35K55.

The first author was partially supported by a grant from the Israel Science Foundation. 
Now let us denote by $S(t)$ the "solution operator" for $(1.1)$, namely, $u(\cdot, t)=$ $S(t) u_{0}$. It is easy to see that the (nonlinear) operator $S(t)$ can be extended continuously to all of $L^{2}\left(\mathbb{R}^{n}\right)$. Indeed, let $u(x, t), v(x, t)$ be two solutions of $(1.1)$, such that $u(x, 0)=u_{0}(x), v(x, 0)=v_{0}(x)$. Then,

$$
(u-v)_{t}=\Delta(u-v)+\mu(|\nabla u|-|\nabla v|) .
$$

Multiplication by $u-v$ and integration over $\mathbb{R}^{n}$ yields, for all $\varepsilon>0$,

$$
\begin{aligned}
& \frac{d}{d t} \int_{\mathbb{R}^{n}}(u-v)^{2} d x \leq-\int_{\mathbb{R}^{n}}|\nabla(u-v)|^{2} d x \\
& \quad+\varepsilon|\mu| \int_{\mathbb{R}^{n}}|\nabla(u-v)|^{2} d x+\frac{|\mu|}{\varepsilon} \int_{\mathbb{R}^{n}}(u-v)^{2} d x,
\end{aligned}
$$

from which we get, in view of Gronwall's inequality,

$$
\left\|S(t) u_{0}-S(t) v_{0}\right\|_{L^{2}\left(\mathbb{R}^{n}\right)} \leq e^{\mu^{2} t}\left\|u_{0}-v_{0}\right\|_{L^{2}\left(\mathbb{R}^{n}\right)}
$$

Observe, however, that (1.4) involves an exponential blow-up in $L^{2}$ norm, which is clearly not "optimal" for smooth, non-negative functions, since we can combine (1.2) and (1.3) to obtain, for such initial functions, a decay in $L^{2}$ norm.

The $L^{1}$ decay result, as expressed in Theorem 1.2, motivates our interest in the possibility of extending $S(t)$ continuously in $L^{1}\left(\mathbb{R}^{n}\right)$ and studying its decay properties for general initial data. This problem is also motivated by some discrete analogs [Sh].

The plan of this paper is as follows.

In Section 2, we prove that the operator $S(t)$ can indeed be extended as a continuous mapping from $L^{1}\left(\mathbb{R}^{n}\right)$ into itself. Furthermore, we obtain some preliminary regularity properties.

In Section 3, we obtain the uniqueness of weak solutions to (1.1) in the class

$$
u(\cdot, t) \in L_{\text {loc }}^{1}\left[\overline{\mathbb{R}}_{+}, W^{1,1}\left(\mathbb{R}^{n}\right)\right] \cap L_{\text {loc }}^{\infty}\left[\mathbb{R}_{+}, L^{1}\left(\mathbb{R}^{n}\right)\right], \mathbb{R}_{+}=(0, \infty),
$$

which, in particular, contains the solutions obtained in Section 2.

In Section 4 we show that the unique solution for (1.1) is indeed a classical solution for $t>0$. Furthermore, the solution is in $C^{2+\alpha, 1+\alpha}\left(\mathbb{R}^{n} \times(\Sigma, T)\right)$ for any $0<\Sigma<T<\infty$.

Sections 5 and 6 are devoted to the $L^{1}$ decay properties of the solutions since $t \rightarrow \infty$. In Section 5 we obtain decay under the assumptions $\mu<0, u_{0}(x) \geq 0$ (equivalently $\mu>0, u_{0}(x) \leq 0$ ). More generally, for $\mu<0$ we show that $u(x, t)^{+} \equiv$ $\max (0, u(x, t))$ decays in $L^{1}\left(\mathbb{R}^{n}\right)$. In Section 6 we construct an example that shows that in general one cannot expect the decay in $L^{1}\left(\mathbb{R}^{n}\right)$ of the solution. The example consists of a smooth nonnegative solution to (1.1) such that its $L^{1}\left(\mathbb{R}^{n}\right)$ norm increases to infinity like $t^{n}$. The example is basically one of an "expanding wave".

We mention that Benachour, Roynette and Vallois [BRV] used probabilistic methods to give asymptotic decay rates in the case that $u$ is spherically symmetric (in $x$ ). It is also interesting to note that the related equation $u_{t}=\Delta u+\mu|\nabla u|^{q}+u^{p}$, $u \geq 0, \mu<0, p, q>1$, has been studied extensively (see, e.g., [AF], [So], [SW] and references there), both in bounded and unbounded domains. Various results concerning existence of global (stationary and nonstationary) solutions, as well as cases where solutions blow up in finite time, have been obtained. However, the case at hand ( $q=1$ and no power of $u$ ) in the whole space is never included. 
In Section 7 we consider the Cauchy problem (1.1) on the torus $T^{n}$, i.e., we treat the case of periodic boundary conditions. We state an existence and uniqueness theorem, the proof of which follows the lines of the one in $\mathbb{R}^{n}$. We then prove that as $t \rightarrow \infty$ the solution tends in $L^{\infty}\left(T^{n}\right)$ to a constant $c\left(u_{0}, \mu\right)$ which is strictly between the essential inf and sup of $u_{0}$. We prove that $c\left(u_{0}, \mu\right) \rightarrow \operatorname{ess} \inf u_{0}$ (resp. ess $\left.\sup u_{0}\right)$ as $\mu \rightarrow-\infty$ (resp. $\mu \rightarrow \infty$ ). Throughout this paper we use \|\|$_{p}$ to denote the $L^{p}\left(\mathbb{R}^{n}\right)$ norm, and $C^{\beta, \gamma}$ for Hölder functions with exponents $\beta, \gamma$ with respect to $x, t$, respectively.

\section{EXISTENCE}

Theorem 2.1. The operator $S(t)$ can be extended, for every $t \geq 0$, as a continuous map of $L^{1}\left(\mathbb{R}^{n}\right)$ into itself.

Furthermore, the solution $u(x, t)=S(t) u_{0}, u_{0} \in L^{1}\left(\mathbb{R}^{n}\right)$, has the following regularity properties,

$$
\begin{gathered}
S(t) u_{0} \in C\left(\overline{\mathbb{R}}_{+}, L^{1}\left(\mathbb{R}^{n}\right)\right), \\
S(t) u_{0} \in L_{\text {loc }}^{1}\left(\overline{\mathbb{R}}_{+}, W^{1,1}\left(\mathbb{R}^{n}\right)\right) \cap L_{\text {loc }}^{\infty}\left(\mathbb{R}_{+}, W^{1,1}\left(\mathbb{R}^{n}\right)\right) .
\end{gathered}
$$

In (2.1), (2.2) we are using the notation $C(I, X)$ to denote continuous functions defined in $I \subseteq \mathbb{R}$ and taking values in the Banach space $X\left(L^{1}(I, X), L^{\infty}(I, X)\right.$ carry analogous meanings). Also, $W^{1,1}\left(\mathbb{R}^{n}\right)$ is the Sobolev space of all $L^{1}$ functions having (distributional) gradients in $L^{1}$.

Proof. Assume first that $u_{0}, v_{0} \in C_{0}^{\infty}\left(\mathbb{R}^{n}\right)$ and let $u(x, t)=S(t) u_{0}, v(x, t)=S(t) v_{0}$ be the corresponding solutions of (1.1). It follows that,

$$
u(x, t)=\int_{\mathbb{R}^{n}} G(x-y, t) u_{0}(y) d y+\mu \int_{0}^{t} \int_{\mathbb{R}^{n}} G(x-y, t-s)|\nabla u(y, s)| d y d s,
$$

where $G(x, t)=(4 \pi t)^{-n / 2} e^{-\frac{|x|^{2}}{4 t}}$ is the heat kernel. Taking the gradient (with respect to $x)$ in (2.3) and subtracting the similar expression for $v(x, t)$ we have, for $t>0$,

$$
\begin{aligned}
\nabla u(x, t) & -\nabla v(x, t)=\int_{\mathbb{R}^{n}} \nabla_{x} G(x-y, t) \cdot\left[u_{0}(y)-v_{0}(y)\right] d y \\
& +\mu \int_{0}^{t} \int_{\mathbb{R}^{n}} \nabla_{x} G(x-y, t-s) \cdot[|\nabla u(y, s)|-|\nabla v(y, s)|] d y d s .
\end{aligned}
$$

It is easily seen (by similarity), that

$$
\int_{\mathbb{R}^{n}}|\nabla G(x, t)| d x=\beta t^{-\frac{1}{2}}, t>0, \beta=\int_{\mathbb{R}^{n}}|\nabla G(x, 1)| d x,
$$

so that, if we denote $B(t)=\|\nabla u-\nabla v\|_{1}, r=\left\|u_{0}-v_{0}\right\|_{1}$, then, by (2.4),

$$
B(t) \leq \beta r t^{-\frac{1}{2}}+\beta|\mu| \int_{0}^{t}(t-s)^{-\frac{1}{2}} B(s) d s .
$$


Set $W(t)=\left[B(t)-\beta r t^{-\frac{1}{2}}\right]^{+}$to obtain,

$$
\begin{aligned}
W(t) & \leq \beta|\mu| \int_{0}^{t}(t-s)^{-\frac{1}{2}}\left[W(s)+\beta r s^{-\frac{1}{2}}\right] d s \\
& =\beta|\mu| \int_{0}^{t}(t-s)^{-\frac{1}{2}} W(s) d s+\pi \beta^{2} r|\mu| .
\end{aligned}
$$

Let $C=C(T)>0$ be so large such that

$$
\beta|\mu| \int_{0}^{T} t^{-\frac{1}{2}} e^{-C t} d t<\frac{1}{2},
$$

then (2.6) yields, for $t \in[0, T]$,

$$
W(t) \leq 2 \pi \beta^{2} r|\mu| e^{C t} .
$$

Turning back to the difference $u-v$ and using (2.3) and the definitions of $r, B(t)$, we get from (2.8),

$$
\begin{aligned}
& \|u(x, t)-v(x, t)\|_{1} \leq\left\|u_{0}-v_{0}\right\|_{1}+|\mu| \int_{0}^{t} B(s) d s \\
& \quad \leq\left\|u_{0}-v_{0}\right\|_{1} \cdot\left\{1+2 \pi \beta^{2} \mu^{2} \cdot \frac{e^{C t}-1}{C}+\beta|\mu| \int_{0}^{t} s^{-\frac{1}{2}} d s\right\},
\end{aligned}
$$

where $0 \leq t \leq T$ and $C=C(T)$ is given by (2.7). This estimate (and the density of $C_{0}^{\infty}\left(\mathbb{R}^{n}\right)$ in $L^{1}\left(\mathbb{R}^{n}\right)$ ) implies that $S(t)$ can be extended continuously to all of $L^{1}\left(\mathbb{R}^{n}\right)$ and that $(2.9)$ is valid for any $u_{0}, v_{0} \in L^{1}\left(\mathbb{R}^{n}\right)$.

Now, recalling the definition of $B(t)$ in $(2.8)$ we have,

$$
\|\nabla u(x, t)-\nabla v(x, t)\|_{1} \leq \beta\left\|u_{0}-v_{0}\right\|_{1} \cdot\left\{t^{-\frac{1}{2}}+2 \pi \beta|\mu| e^{C t}\right\}
$$

for $0<t \leq T$. The estimate $(2.10)$ yields readily $S(t) u_{0} \in L_{\text {loc }}^{\infty}\left(\mathbb{R}_{+}, W^{1,1}\left(\mathbb{R}^{n}\right)\right) \cap$ $L_{\text {loc }}^{1}\left(\overline{\mathbb{R}}_{+}, W^{1,1}\left(\mathbb{R}^{n}\right)\right)$, and also the validity of $(2.3)$ for a general $u_{0} \in L^{1}\left(\mathbb{R}^{n}\right)$. Finally,

$$
\begin{aligned}
S(t+\Delta t) u_{0}-S(t) u_{0}=\int_{\left(\mathbb{R}^{n}\right)}(G(x-y, t & +\Delta t)-G(x-y, t)) u_{0}(y) d y \\
& +\mu \int_{t} \int_{\mathbb{R}^{n}}^{t+\Delta t} G(x-y, t-s)|\nabla u(y, s)| d y d s,
\end{aligned}
$$

so that,

$$
\begin{aligned}
\| S(t & +\Delta t) u_{0}-S(t) u_{0} \|_{1} \\
& \leq\left\|u_{0}\right\|_{1} \cdot \int_{\left(\mathbb{R}^{n}\right)}|G(x, t+\Delta t)-G(x, t)| d x+|\mu| \int_{t}^{t+\Delta t}\|\nabla u(\cdot, s)\|_{1} d s,
\end{aligned}
$$


which, combined with the above observations, yields,

$$
S(t) u_{0} \in C\left(\overline{\mathbb{R}}_{+}, L^{1}\left(\mathbb{R}^{n}\right)\right) .
$$

This concludes the proof of the theorem.

Remark 2.2. It follows actually from the proof that $S(t) u_{0} \in C\left(\mathbb{R}_{+}, W^{1,1}\left(\mathbb{R}^{n}\right)\right)$ for $u_{0} \in L^{1}\left(\mathbb{R}^{n}\right)$.

\section{UNIQUENESS}

Theorem 3.1. Let $u(x, t)$ be a distribution solution to the Cauchy problem (1.1), where

$$
\begin{aligned}
& u(\cdot, t) \in L_{\text {loc }}^{1}\left(\overline{\mathbb{R}}_{+}, W^{1,1}\left(\mathbb{R}^{n}\right)\right) \\
& \quad \cap L_{\text {loc }}^{\infty}\left(\mathbb{R}_{+}, L^{1}\left(\mathbb{R}^{n}\right)\right), u(\cdot, t) \underset{t \rightarrow 0}{\longrightarrow} u_{0} \quad \text { in } \quad L^{1}\left(\mathbb{R}^{n}\right) .
\end{aligned}
$$

Let

$$
G(x, t)=(4 \pi t)^{-n / 2} e^{-\frac{|x|^{2}}{4 t}}, t>0,
$$

be the heat kernel. Then,

$$
u(x, t)=\int_{\mathbb{R}^{n}} G(x-y, t) u_{0}(y) d y+\mu \int_{0}^{t} \int_{\mathbb{R}^{n}} G(x-y, t-s)|\nabla u(y, s)| d y d s .
$$

Proof. Let

$$
v(x, t)=\mu \int_{0}^{t} \int_{\mathbb{R}^{n}} G(x-y, t-s)|\nabla u(y, s)| d y d s .
$$

We claim that as a function of $t, v(\cdot, t)$ is a well defined function in $C\left(\overline{\mathbb{R}}_{+}, L^{1}\left(\mathbb{R}^{n}\right)\right)$. Indeed, since $\int_{\mathbb{R}^{n}} G(x, t) d x=1$ for $t>0$, we have

$$
\left\|\int_{\mathbb{R}^{n}} G(\cdot-y, t-s)|\nabla u(y, s)| d y\right\|_{1}=\|\nabla u(\cdot, s)\|_{1} .
$$

From (3.1) we know that $\|\nabla u(\cdot, s)\|_{1} \in L_{\text {loc }}^{1}\left(\mathbb{R}_{+}\right)$. Thus, using Fubini's theorem we have

$$
\begin{aligned}
& \int_{\mathbb{R}^{n}}|v(x, t)| d x=|\mu| \int_{\mathbb{R}^{n}} \int_{0}^{t} \int_{\mathbb{R}^{n}} G(x-y, t-s)|\nabla u(y, s)| d y d s d x \\
& =|\mu| \int_{0}^{t}\left\|\int_{\mathbb{R}^{n}} G(\cdot-y, t-s)|\nabla u(y, s)| d y\right\|_{1} d s=|\mu| \int_{0}^{t}\|\nabla u(\cdot, s)\|_{1} d s<\infty .
\end{aligned}
$$

Furthermore, it is easy to see, by standard arguments, that the equation

$$
v_{t}=\Delta v+\mu|\nabla u|
$$

is satisfied in the sense of distributions.

Since $v$ satisfies (3.5) it follows that $w \equiv u-v$ satisfies

$$
w_{t}=\Delta w, \quad w(\cdot, t) \underset{t \rightarrow 0}{\rightarrow} u_{0} \quad \text { in } \quad L^{1}\left(\mathbb{R}^{n}\right),
$$


in the sense of distributions, where $w \in L_{\text {loc }}^{\infty}\left(\mathbb{R}_{+}, L^{1}\left(\mathbb{R}^{n}\right)\right)$. Let

$$
z(x, t)=\int_{\mathbb{R}^{n}} G(x-y, t) u_{0}(y) d y .
$$

By Young's inequality $\|z(\cdot, t)\|_{1} \leq\left\|u_{0}\right\|_{1}$ for any $t>0$. Furthermore, $z$ satisfies the heat equation in the sense of distributions in $\mathbb{R}^{n} \times \mathbb{R}_{+}$, and $z(\cdot, t) \rightarrow u_{0}(\cdot)$ in $L^{1}\left(\mathbb{R}^{n}\right)$ as $t \rightarrow 0$. This follows from the fact that $\left\|G * u_{0}-u_{0}\right\|_{1} \leq 2\left\|u_{0}\right\|_{1}$ and that for smooth $u_{0}, G * u_{0} \underset{t \rightarrow 0}{\rightarrow} u_{0}$ pointwise.

We conclude that $Q \equiv w-z$ satisfies $Q_{t}=\Delta Q$ in the sense of distributions, where $Q \in L_{\text {loc }}^{\infty}\left(\mathbb{R}^{+}, L^{1}\left(\mathbb{R}^{n}\right)\right)$ and $Q(\cdot, t) \underset{t \rightarrow 0}{\rightarrow} 0$ in $L^{1}\left(\mathbb{R}^{n}\right)$.

It thus follows that $Q \equiv 0$, e.g., since $Q_{\varepsilon} \equiv \delta_{\varepsilon} * Q$ is smooth and satisfies $\partial_{t} Q_{\varepsilon}=\Delta Q_{\varepsilon}$ and $Q_{\varepsilon}(\cdot, t) \underset{t \rightarrow 0}{\rightarrow} 0$.

Corollary 3.2. Let $u_{0} \in L^{1}\left(\mathbb{R}^{n}\right)$. Then there exists a unique solution to the Cauchy problem (1.1) in the class of functions (3.1).

Proof. The existence of such a solution was proved in Theorem 2.1. We need to show its uniqueness. Let $u_{1}, u_{2}$ be two solutions satisfying the assumptions. By Theorem 3.1,

$$
u_{1}(x, t)-u_{2}(x, t)=\mu \int_{0}^{t} \int_{\mathbb{R}^{n}} G(x-y, t-s)\left[\left|\nabla u_{1}(y, s)\right|-\left|\nabla u_{2}(y, s)\right|\right] d y d s,
$$

hence

$$
\nabla u_{1}(x, t)-\nabla u_{2}(x, t)=\mu \int_{0}^{t} \int_{\mathbb{R}^{n}} \nabla G(x-y, t-s)\left[\left|\nabla u_{1}(y, s)\right|-\left|\nabla u_{2}(y, s)\right|\right] d y d s .
$$

As in the derivation of $(2.5)$

$$
\left\|\nabla\left(u_{1}-u_{2}\right)(\cdot, t)\right\|_{1} \leq|\mu| \int_{0}^{t}(t-s)^{-1 / 2}\left\|\nabla\left(u_{1}-u_{2}\right)(\cdot, s)\right\|_{1} \quad d s,
$$

from which $\left\|\nabla\left(u_{1}-u_{2}\right)(\cdot, t)\right\|_{1} \equiv 0$ follows, implying $u_{1}=u_{2}$ a.e. by (3.6).

Remark 3.3. The uniqueness result ensures that any weak solution satisfying (3.1) is the solution obtained in Theorem 2.1 and thus satisfies the estimates in the proof of that theorem.

\section{Regularity}

Theorem 4.1. Let $u(x, t)$ be a distribution solution to the Cauchy problem (1.1), where

$$
u(\cdot, t) \in L_{\mathrm{loc}}^{1}\left(\overline{\mathbb{R}}_{+}, W^{1,1}\left(\mathbb{R}^{n}\right)\right) \cap L_{\mathrm{loc}}^{\infty}\left(\mathbb{R}_{+}, L^{1}\left(\mathbb{R}^{n}\right)\right)
$$

Then

$$
u \in C^{2+\alpha, 1+\alpha}\left(\mathbb{R}^{n} \times(\varepsilon, T)\right)
$$

for any $0<\alpha<1,0<\varepsilon<T<\infty$.

The proof of the theorem will follow from the following lemmas. 
Lemma 4.2. Let $u$ satisfy the conditions of the theorem, then

$$
\nabla u(\cdot, t) \in L_{\text {loc }}^{\infty}\left(\mathbb{R}_{+}, L^{\infty}\left(\mathbb{R}^{n}\right)\right) .
$$

Proof. We first need some $L^{p}\left(\mathbb{R}^{n}\right)$ properties of the heat kernel (3.2). By scaling

$$
G(x, t)=\frac{1}{t^{n / 2}} G\left(\frac{x}{\sqrt{t}}, 1\right) .
$$

Interpolating, using the scaling and the properties of $G$, we have

$$
\|G(\cdot, t)\|_{p} \leq\|G(\cdot, t)\|_{\infty}^{\frac{p-1}{p}}\|G(\cdot, t)\|_{1}^{1 / p}=c t^{-\frac{n}{2} \frac{p-1}{p}} .
$$

Using the scaling again, we obtain that, in fact, for some $c>0$,

$$
\|G(\cdot, t)\|_{p}=c \cdot t^{-\frac{n}{2} \cdot \frac{p-1}{p}} .
$$

Now

$$
\begin{aligned}
\int_{\mathbb{R}^{n}}|\nabla G(x, t)|^{p} d x & =\frac{1}{t^{\left(\frac{n}{2}+\frac{1}{2}\right) p}} \int_{\mathbb{R}^{n}}\left|\nabla G\left(\frac{x}{\sqrt{t}}, 1\right)\right|{ }^{p} d x=\frac{1}{t^{\frac{n+1}{2} p}} \int_{\mathbb{R}^{n}}|\nabla G(y, 1)|^{p} t^{n / 2} d y \\
& =c_{1} t^{\frac{n}{2}-\frac{n+1}{2} p}
\end{aligned}
$$

where $c_{1}=\int_{\mathbb{R}^{n}}|\nabla G(y, 1)|^{p} d y<\infty$.

We conclude that $\|G(\cdot, t)\|_{p}$ is integrable on any interval $(0, T), T<\infty$, if $-\frac{n}{2}\left(1-\frac{1}{p}\right)>-1$, i.e. $p<1+\frac{2}{n-2}$, and $\|\nabla G(\cdot, t)\|_{p}$ is integrable on such intervals if $p<1+\frac{1}{n-1}$.

We will also need an estimate of $\|\nabla u(\cdot, t)\|_{L^{1}\left(\mathbb{R}^{n}\right)}$ for $\varepsilon \leq t \leq T$ where $\varepsilon>0$ is small and $T<\infty$. To obtain such an estimate we go back to the proof of Theorem 2.1 .

In view of Remark 3.3 the estimate (2.9) is valid for the class of weak solutions considered here. Let $v_{0}(x) \equiv 0$ so that $v(x, t) \equiv 0$. Then (2.9) yields

$$
\|u(\cdot, t)\|_{1} \leq C_{3}(T) \cdot\left\|u_{0}\right\|_{1} .
$$

Plugging this into (2.8), where now $r=\left\|u_{0}\right\|_{1}$ and

$$
W(t)=\left[\|\nabla u(\cdot, t)\|_{1}-\beta\left\|u_{0}\right\|_{1} \cdot t^{-1 / 2}\right]^{+},
$$

we get

$$
\|\nabla u(\cdot, t)\|_{1} \leq C_{4}(T)\left[t^{-1 / 2}+e^{c t}\right]\left\|u_{0}\right\|_{1} .
$$

Thus

$$
\|\nabla u(\cdot, t)\|_{1} \leq C_{5}(T)\left\|u_{0}\right\|_{1} \cdot t^{-1 / 2}, \quad 0<t \leq T .
$$

From the representation (3.3) (and Corollary 3.2) it is clear that for $t>\varepsilon>0$,

$$
u(x, t)=\int_{\mathbb{R}^{n}} G(x-y, t-\varepsilon) u(y, \varepsilon) d y+\mu \int_{\varepsilon}^{t} \int_{\mathbb{R}^{n}} G(x-y, t-s)|\nabla u(y, s)| d y d s,
$$


and therefore

$$
\nabla u(x, t)=\int_{\mathbb{R}^{n}} G(x-y, t-\varepsilon) \nabla u(y, \varepsilon) d y+\mu \int_{\varepsilon}^{t} \int_{\mathbb{R}^{n}} \nabla G(x-y, t-s)|\nabla u(y, s)| d y d s .
$$

By Young's inequality, (4.2) and (4.4), it follows that

$$
\begin{aligned}
& \left\|\int_{\mathbb{R}^{n}} G(\cdot-y, t-\varepsilon) \nabla u(y, \varepsilon) d y\right\|_{1+\frac{1}{n}} \\
& \quad \leq\|G(\cdot, t-\varepsilon)\|_{1+\frac{1}{n}}\|\nabla u(\cdot, \varepsilon)\|_{1} \leq C_{6}(T) \varepsilon^{-1 / 2}(t-\varepsilon)^{-\frac{1}{2} \frac{n}{n+1}}\left\|u_{0}\right\|_{1} .
\end{aligned}
$$

Young's inequality and (4.3) give also,

$$
\begin{aligned}
\left\|\int_{\mathbb{R}^{n}} \nabla G(\cdot-y, t-s)|\nabla u(y, s)| d y\right\|_{1+\frac{1}{n}} & \leq\|\nabla G(\cdot, t-s)\|_{1+\frac{1}{n}} \cdot\|\nabla u(\cdot, s)\|_{1} \\
& \leq c_{1}(t-s)^{-\frac{2 n+1}{2 n+2}}\|\nabla u(\cdot, s)\|_{1} .
\end{aligned}
$$

Thus, in view of (4.4),

$$
\begin{aligned}
& \left\|\int_{\varepsilon} \int_{\mathbb{R}^{n}} \nabla G(\cdot-y, t-s)|\nabla u(y, s)| d y d s\right\|_{1+\frac{1}{n}} \\
& \quad \leq \int_{\varepsilon}^{t}\left\|\int_{\mathbb{R}^{n}} \nabla G(\cdot-y, t-s)|\nabla u(y, s)| d y\right\|_{1+\frac{1}{n}} d s \\
& \quad \leq C_{7}(T) \int_{\varepsilon / t}^{1}(1-\theta)^{-\frac{2 n+1}{2 n+2}} \theta^{-1 / 2} d \theta \cdot t^{-\frac{n}{2 n+2}}\left\|u_{0}\right\|_{1} \\
& \quad \leq C_{7}(T)\left\|u_{0}\right\|_{1} B\left(\frac{1}{2 n+2}, \frac{1}{2}\right) \cdot t^{-\frac{n}{2(n+1)}} \leq C_{8}(T)\left\|u_{0}\right\|_{1} \cdot \varepsilon^{-1 / 2} .
\end{aligned}
$$

Using (4.7) and (4.9) in (4.6) we obtain

$$
\begin{aligned}
\|\nabla u(\cdot, t)\|_{1+\frac{1}{n}} & \leq C_{9}(T)\left\|u_{0}\right\|_{1} \cdot \varepsilon^{-1 / 2}\left[(t-\varepsilon)^{-\frac{1}{2} \frac{n}{n+1}}+1\right] \\
& \leq C_{10}(T)\left\|u_{0}\right\|_{1} \cdot \varepsilon^{-1 / 2}(t-\varepsilon)^{-1 / 2} .
\end{aligned}
$$

We may now repeat the procedure, with $\varepsilon$ replaced by $2 \varepsilon$ in (4.5) and (4.6). Then we have

$$
\begin{gathered}
\left\|\int_{\mathbb{R}^{n}} G(\cdot-y, t-2 \varepsilon) \nabla u(y, 2 \varepsilon) d y\right\|_{1+\frac{2}{n-1}} \leq\|G(\cdot, t-2 \varepsilon)\|_{1+\frac{1}{n}}\|\nabla u(\cdot, 2 \varepsilon)\|_{1+\frac{1}{n}} \\
\leq(t-2 \varepsilon)^{-\frac{1}{2} \frac{n}{n+1}} C_{10}(T)\left\|u_{0}\right\|_{1} \cdot \varepsilon^{-1} \\
\left\|\int_{\mathbb{R}^{n}} \nabla G(\cdot-y, t-s)|\nabla u(y, s)| d y\right\|_{1+\frac{2}{n-1}} \\
\leq\|\nabla G(\cdot, t-s)\|_{1+\frac{1}{n}} \cdot\|\nabla u(\cdot, s)\|_{1+\frac{1}{n}} \\
\leq c_{1}(t-s)^{-\frac{2 n+1}{2 n+2}} C_{10}(T)\left\|u_{0}\right\|_{1} \varepsilon^{-1 / 2}(s-\varepsilon)^{-1 / 2}
\end{gathered}
$$




$$
\begin{aligned}
& \left\|\int_{2 \varepsilon}^{t} \int_{\mathbb{R}^{n}} \nabla G(x-y, t-s)|\nabla u(y, s)| d y d s\right\|_{1+\frac{2}{n-1}} \\
& \quad \leq \int_{2 \varepsilon}^{t}\left\|\int_{\mathbb{R}^{n}} \nabla G(\cdot-y, t-s)|\nabla u(y, s)| d y\right\|_{1+\frac{2}{n-1}} d s \\
& \quad \leq C_{11}(T) \cdot \int_{2 \varepsilon}^{t}(t-s)^{-\frac{2 n+1}{2 n+2}}(s-\varepsilon)^{-1 / 2} d s \cdot\left\|u_{0}\right\|_{1} \varepsilon^{-1 / 2} \leq C_{12}(T)\left\|u_{0}\right\|_{1} \varepsilon^{-1},
\end{aligned}
$$

so that for $T \geq t>2 \varepsilon$,

$$
\begin{aligned}
\|\nabla u(\cdot, t)\|_{1+\frac{2}{n-1}} & \leq C_{12}(T)\left\|u_{0}\right\|_{1} \cdot \varepsilon^{-1}\left[(t-2 \varepsilon)^{-\frac{1}{2} \frac{n}{n+1}}+1\right] \\
& \leq C_{13}(T)\left\|u_{0}\right\|_{1} \varepsilon^{-1}(t-2 \varepsilon)^{-1 / 2} .
\end{aligned}
$$

It is clear now how this process is continued $n$ times. As above we get

$$
\|\nabla u(\cdot, t)\|_{\infty} \leq C(\varepsilon, T)\left\|u_{0}\right\|_{1} \quad \text { for } \quad T \geq t \geq(n+1) \varepsilon .
$$

Lemma 4.3. If $u$ satisfies the conditions of the theorem, then for any $0<\varepsilon<T<$ $\infty$ and $0<\alpha<1$,

$$
\nabla u \in C^{\alpha, \alpha / 2}\left((\varepsilon, T) \times \mathbb{R}^{n}\right) .
$$

Proof. Fix $0<\varepsilon<T$. For $\varepsilon<t<T$ and $\eta_{i}=\left(0, \ldots, \Delta x_{i}, 0\right)$.

$$
\begin{aligned}
& \nabla u\left(x+\eta_{i}, t\right)-\nabla u(x, t)=\int_{\mathbb{R}^{n}}\left[G\left(x+\eta_{i}-y, t-\varepsilon\right)-G(x-y, t-\varepsilon)\right] \nabla u(y, \varepsilon) d y \\
& +\mu \int_{\varepsilon}^{t} \int_{\mathbb{R}^{n}}\left[\nabla G\left(x+\eta_{i}-y, t-s\right)-\nabla G(x-y, t-s)\right]|\nabla u(y, s)| d y d s .
\end{aligned}
$$

Now

$$
\begin{aligned}
& \int_{\mathbb{R}^{n}}\left|G\left(x+\eta_{i}, t\right)-G(x, t)\right| d x=\left|\eta_{i}\right| \int_{\mathbb{R}^{n}}\left|\int_{0}^{1} \frac{\partial G}{\partial x_{i}}\left(x+\sigma \eta_{i}, t\right) d \sigma\right| d x \\
& \leq\left|\eta_{i}\right| \cdot \int_{\mathbb{R}^{n}}\left|\frac{\partial G}{\partial x_{i}}(x, t)\right| d x \leq \frac{C}{\sqrt{t}}\left|\eta_{i}\right|, \\
& \int_{\mathbb{R}^{n}}\left|\nabla G\left(x+\eta_{i}, t\right)-\nabla G(x, t)\right| d x=\left|\eta_{i}\right| \int_{\mathbb{R}^{n}}\left|\int_{0}^{1} \frac{\partial(\nabla G)}{\partial x_{i}}\left(x+\sigma \eta_{i}, t\right) d \sigma\right| d x \\
& \leq\left|\eta_{i}\right| \cdot \int_{\mathbb{R}^{n}}\left|\frac{\partial \nabla G}{\partial x_{i}}(x, t)\right| d x \leq\left|\eta_{i}\right| \cdot C t^{-1} .
\end{aligned}
$$


We can write, for $0<\alpha<1$,

$$
\begin{aligned}
& \int_{\mathbb{R}^{n}}\left|\nabla G\left(x+\eta_{i}, t\right)-\nabla G(x, t)\right| d x \leq\left(\left|\eta_{i}\right| \cdot C t^{-1}\right)^{\alpha} \cdot\left(2 \int_{\mathbb{R}^{n}}|\nabla G(x, t)| d x\right)^{1-\alpha} \\
& =C\left|\eta_{i}\right|^{\alpha} t^{-\alpha-\frac{1}{2}(1-\alpha)}=C\left|\eta_{i}\right|^{\alpha} t^{-\frac{1}{2}(1+\alpha)}
\end{aligned}
$$

Inserting (4.16) and (4.18) in (4.15) we get, for $2 \varepsilon<t<T$,

$$
\begin{aligned}
& \left|\nabla u\left(x+\eta_{i}, t\right)-\nabla u(x, t)\right| \\
& \quad \leq C|\mu|\left|\eta_{i}\right|^{\alpha}\left[\left|\eta_{i}\right|^{1-\alpha} \varepsilon^{-1 / 2}+(t-\varepsilon)^{\frac{1}{2}(1-\alpha)}\right]\|\nabla u\|_{L^{\infty}\left((\varepsilon, T) \times \mathbb{R}^{n}\right)} .
\end{aligned}
$$

Thus it follows that $\nabla u(x, t)$ is Hölder continuous with any exponent $\alpha<1$ in $x$ in any strip $(\varepsilon, T) \times \mathbb{R}^{n}, \quad 0<\varepsilon<T<\infty$.

Next we prove that $\nabla u(x, t)$ is Hölder continuous in $t$. By (3.3)

$$
\begin{aligned}
\nabla u(x, t+\Delta t)-\nabla u(x, t) & =\int_{\mathbb{R}^{n}} G(x-y, \Delta t)[\nabla u(y, t)-\nabla u(x, t)] d y \\
& +\mu \int_{0}^{\Delta t} \int_{\mathbb{R}^{n}} \nabla G(x-y, \Delta t-s)|\nabla u(y, t+\Delta t-s)| d y d s .
\end{aligned}
$$

The second term on the right can be estimated by $C(\Delta t)^{1 / 2}\|\nabla u\|_{L^{\infty}\left([t, t+\Delta t] \times \mathbb{R}^{n}\right)}$ as before. To estimate the first term we use

$$
|\nabla u(y, t)-\nabla u(x, t)| \leq C \max \left\{|x-y|^{\alpha},|x-y|\right\}\|\nabla u\|_{L^{\infty}\left((\varepsilon, T) \times \mathbb{R}^{n}\right)}
$$

for any $0<\alpha<1$ (where $C=C(\alpha, \varepsilon, T)$ ), so

$$
\begin{aligned}
& \left|\int_{\mathbb{R}^{n}} G(x-y, \Delta t)[\nabla u(y, t)-\nabla u(x, t)] d y\right| \\
& \leq \int_{\mathbb{R}^{n}}(\Delta t)^{-n / 2} G\left(\frac{x-y}{\sqrt{\Delta t}}, 1\right) \cdot|\nabla u(y, t)-\nabla u(x, t)| d y \\
& \leq C \int_{|x-y| \leq 1}(\Delta t)^{-n / 2} G\left(\frac{x-y}{\sqrt{\Delta t}}, 1\right)\left(\frac{|x-y|}{\sqrt{\Delta t}}\right)^{\alpha} \sqrt{\Delta t}{ }^{\alpha} d y \\
& \quad+C \int_{|x-y|>1}(\Delta t)^{-n / 2} G\left(\frac{x-y}{\sqrt{\Delta t}}, 1\right) \frac{|x-y|}{\sqrt{\Delta t}} \sqrt{\Delta t} d y \\
& \leq\left[(\Delta t)^{\alpha / 2}+(\Delta t)^{1 / 2}\right] \cdot \int_{\mathbb{R}^{n}} G(z, 1) \max \left\{|z|,|z|^{\alpha}\right\} d z=C\left[(\Delta t)^{\alpha / 2}+(\Delta t)^{1 / 2}\right]
\end{aligned}
$$

where $C$ depends on $\|\nabla u\|_{L^{\infty}\left((\varepsilon, T) \times \mathbb{R}^{n}\right)}$. Thus, we conclude that

$$
\nabla u \in C^{\alpha, \frac{\alpha}{2}}\left((\varepsilon, T) \times \mathbb{R}^{n}\right)
$$

for any $0<\alpha<1$ and any $0<\varepsilon<T<\infty$.

Theorem 4.1 now follows from the next theorem, the proof of which can be found in Friedman [F] (Theorem 10, p. 72). 
Theorem 4.4. Let $u$ be a solution of

$$
u_{t}-\Delta u=f
$$

where $f \in C^{\alpha, \frac{\alpha}{2}}$, then $u, u_{x_{i}}, u_{t}$ and $u_{x_{i} x_{j}}, i, j=1, \cdot, n$, are all Hölder continuous with exponent $\alpha$ in $x$ and exponent $\frac{\alpha}{2}$ in $t$.

Corollary 4.5. If $u$ satisfies the assumptions of Theorem 4.1, then $u$ is a classical solution of (1.1). It also satisfies

$$
u(\cdot, t) \in L^{\infty}\left([\varepsilon, \infty), W^{1, \infty}\left(\mathbb{R}^{n}\right)\right), \text { for any } \varepsilon>0 .
$$

Furthermore,

$$
\begin{gathered}
\|u\|_{L^{\infty}\left(\mathbb{R}^{n} \times[\varepsilon, \infty)\right)}=\|u(\cdot, \varepsilon)\|_{\infty}<\infty, \\
\|\nabla u\|_{L^{\infty}\left(\mathbb{R}^{n} \times[\varepsilon, \infty)\right)} \leq\|\nabla u(\cdot, \varepsilon)\|_{\infty}<\infty .
\end{gathered}
$$

Proof. It is clear that $\|u(\cdot, \varepsilon)\|_{\infty}<\infty$, since we know that $\left\|u\left(\cdot, \frac{\varepsilon}{2}\right)\right\|_{1}<\infty$ and that $\|\nabla u(\cdot, \varepsilon)\|_{\infty}<\infty$. Theorem 4.1 implies that $u$ is a classical solution and thus the maximum principle may be applied to obtain (4.19) (see [B]).

We now wish to show that $\left\|u_{x_{i}}\right\|_{L^{\infty}\left(\mathbb{R}^{n} \times[\varepsilon, \infty)\right)} \leq\left\|u_{x_{i}}(\cdot, \varepsilon)\right\|_{\infty}$.

Let $v(x, t)$ be the solution to (1.1) with initial conditions $v_{0}(x)=u(x, \varepsilon)$. By the semigroup property, implied by uniqueness, $v(x, t)=u(x, t+\varepsilon)$, and thus it is enough to show that $\left\|v_{x_{i}}\right\|_{L^{\infty}\left(\mathbb{R}^{n} \times \mathbb{R}_{+}\right)} \leq\left\|v_{x_{i}}(\cdot, 0)\right\|_{\infty}$. Let $\left\{v_{0}^{(k)}\right\}_{k=1}^{\infty} \subseteq C_{0}^{\infty}\left(\mathbb{R}^{n}\right)$ such that $v_{0}^{(k)} \rightarrow v_{0}$ in $L^{1}\left(\mathbb{R}^{n}\right)$ and $\left\|\frac{\partial}{\partial x_{i}} v_{0}^{(k)}\right\|_{\infty} \leq\left(1+\frac{1}{k}\right)\left\|\frac{\partial}{\partial x_{i}} v_{0}\right\|_{\infty}$. According to $(2.10)$ there exists a subsequence $\left\{k_{j}\right\}_{j=1}^{\infty}$ such that $\nabla v_{0}^{\left(k_{j}\right)}(x) \rightarrow \nabla v_{0}(x)$ and $\nabla v^{\left(k_{j}\right)}(x, t) \rightarrow \nabla v(x, t)$ a.e. in $x$ for each $t>0$. The maximum principle stated in Theorem 1.1 is valid for all $\frac{\partial}{\partial x_{i}} \nabla v^{\left(k_{j}\right)}$ and thus the conclusion follows.

\section{Decay for Large Time}

Theorem 5.1. Let $\mu<0$ and $0 \leq u_{0} \in L^{1}\left(\mathbb{R}^{n}\right)$. Then, for every $t \geq 0, u(x, t)=$ $S(t) u_{0} \geq 0$ and,

$$
\int_{\mathbb{R}^{n}} u(x, t) d x \text { decreases to zero as } t \rightarrow+\infty .
$$

Furthermore, with $\mu<0$, let $v_{0} \in L^{1}\left(\mathbb{R}^{n}\right)$ be not necessarily positive, and let $v(x, t)=S(t) v_{0}$. Then

$$
\int_{\mathbb{R}^{n}} v^{+}(x, t) d x \rightarrow 0 \quad \text { as } \quad t \rightarrow \infty .
$$

(We are using the notation $\phi^{+}=\max (\phi(x), 0)$.)

Proof. Let $0 \leq u_{0} \in L^{1}\left(\mathbb{R}^{n}\right)$ and $\mu<0$. Using the maximum principle for (1.1) (Theorem 1.1) as well as the convergence argument in the proof of Corollary 4.5, we see that $S(t) u_{0} \geq 0$ for all $t \geq 0$. Integrating (2.3) with respect to $x$ and letting $t \rightarrow+\infty$ we get,

$$
I(t)=\int_{\mathbb{R}^{n}} u(x, t) d x \text { is decreasing in } \quad t
$$




$$
\begin{gathered}
\int_{0}^{\infty} \int_{\mathbb{R}^{n}}|\nabla u(x, t)| d x d t<\infty, \\
u(x, t) \leq \int_{\mathbb{R}^{n}} G(x-y, t) u_{0}(y) d y \equiv \tilde{u}(x, t),(x, t) \in \mathbb{R}^{n} \times(0, \infty) .
\end{gathered}
$$

Let $\varepsilon>0$. It follows from (5.2) that there exists a sequence $0<t_{1}<t_{2}<\cdots<$ $t_{m}<\cdots \rightarrow+\infty$ such that

$$
\int_{\mathbb{R}^{n}}\left|\nabla u\left(x, t_{j}\right)\right| d x<\varepsilon t_{j}^{-1}, \quad j=1,2, \ldots
$$

Applying the Sobolev inequality [GT] we get, with some constant $C=C(n)$,

$$
\int_{|x| \leq t_{j}} u\left(x, t_{j}\right)^{\frac{n}{n-1}} d x \leq\left(C \varepsilon t_{j}^{-1}\right)^{\frac{n}{n-1}}
$$

(we are assuming $n>1$. The case $n=1$ is simpler). Invoking Hölder's inequality leads to

$$
\int_{|x| \leq t_{j}} u\left(x, t_{j}\right) d x \leq C \varepsilon \omega_{n}^{1 / n} .
$$

$\left(\omega_{n}=\right.$ volume of the unit ball in $\left.\mathbb{R}^{n}\right)$. On the other hand, the solution $\tilde{u}(x, t)$ of the heat equation satisfies

$$
\int_{|x| \geq t_{j}} \tilde{u}\left(x, t_{j}\right) d x \underset{j \rightarrow \infty}{\rightarrow} 0
$$

so that, by (5.3),

$$
\int_{|x| \geq t_{j}} u\left(x, t_{j}\right) d x \underset{j \rightarrow \infty}{\rightarrow} 0 .
$$

Combining (5.5) and (5.6) we have $I\left(t_{j}\right) \rightarrow 0$ as $j \rightarrow \infty$, which concludes the proof of the theorem in the case that $u_{0} \geq 0$.

Now let $v_{0} \in L^{1}\left(\mathbb{R}^{n}\right)$. Taking the positive part in (2.3) and noting that $\left|\nabla \phi^{+}\right|=$ $\operatorname{sgn} \phi^{+} \cdot|\nabla \phi|$ for every $\phi \in W^{1,1}\left(\mathbb{R}^{n}\right)$, we have

$$
v^{+}(x, t) \leq \int_{\mathbb{R}^{n}} G(x-y, t) v_{0}^{+}(y) d y-|\mu| \int_{0}^{t} \int_{\mathbb{R}^{n}} G(x-y, t-s)\left|\nabla v^{+}(y, s)\right| d y d s \equiv J(x, t) .
$$

As in the first part of the proof we conclude that $\int_{\mathbb{R}^{n}} J(x, t) d x \rightarrow 0$ as $t \rightarrow \infty$.

\section{6. $L^{1}$ Growth}

In this section we show that in contrast to the result of Theorem 5.1, if $u \geq 0$ and $\mu>0$ (which is equivalent by the change $u \rightarrow-u$ to $u \leq 0, \mu<0$ ), $\|u(\cdot, t)\|_{1}$ can grow to infinity as $t \rightarrow \infty$. The idea is that for some $u_{0}(x)$, suitably concentrated around $x=0$, the solution $u(x, t)$ represents an expanding profile. 
Theorem 6.1. There exists a smooth, rapidly decaying function $u_{0}(x)$, such that the classical solution $u$ of

$$
u_{t}=\Delta u+|\nabla u|, \quad u(x, 0)=u_{0}(x), \quad x \in \mathbb{R}^{n},
$$

satisfies

$$
\|u(\cdot, t)\|_{1}>c t
$$

for some $c>0$.

Lemma 6.2. Let $u$ satisfy (6.1), and let $v$ satisfy $v_{t}=\Delta v+|\nabla v|+f, \quad v(x, 0)=$ $v_{0}(x)$, where $|f(x, t)| \leq \alpha(t)$. Suppose further that $u, v$ decay to zero as $|x| \rightarrow \infty$, uniformly on finite time intervals. Then

$$
\inf \left(v_{0}-u_{0}\right)-\int_{0}^{t} \alpha(s) d s \leq v(x, t)-u(x, t) \leq \sup \left(v_{0}-u_{0}\right)+\int_{0}^{t} \alpha(s) d s .
$$

Proof (of the lemma). We have

$$
(v-u)_{t}=\Delta(v-u)+[|\nabla v|-|\nabla u|]+f,
$$

so that,

$$
\left(v-u-\int_{0}^{t} \alpha(x) d s\right)_{t} \leq \Delta(v-u)+[|\nabla v|-|\nabla u|] .
$$

Let $w=v-\int_{0}^{t} \alpha(s) d s$. Then,

$$
(w-u)_{t} \leq \Delta(w-u)+[|\nabla w|-|\nabla u|],
$$

from which it follows that $w-u$ cannot attain a maximum for $t>0$. To see this pick any $\beta>0$. Then,

$$
(w-u-\beta t)_{t} \leq \Delta(w-u-\beta t)+[|\nabla(w-\beta t)|-|\nabla u|]-\beta .
$$

Suppose $w-u-\beta t$ has a maximum at a point $\left(x_{0}, t_{0}\right)$, then the left hand side is nonnegative while $\Delta(w-u-\beta t)\left(x_{0}, t_{0}\right) \leq 0, \quad \nabla(w-u-\beta t)=0 \Rightarrow|\nabla(w-\beta t)|-$ $|\nabla u|=0$, so the right hand side is negative, which is a contradiction. Observe now that $\lim _{|x| \rightarrow \infty}[w(x, t)-u(x, t)-\beta t]=\lim _{|x| \rightarrow \infty}\left[v(x, t)-u(x, t)-\int_{0}^{t} \alpha(s) d s-\beta t\right]$ $=-\int_{0}^{t} \alpha(s) d s-\beta t<0$, so that $\sup _{x}[w(x, t)-u(x, t)-\beta t] \leq \sup \left(v_{0}-u_{0}\right)$. (Note that $\left.\sup \left(v_{0}-u_{0}\right) \geq 0\right)$. Letting $\stackrel{x}{\beta} \rightarrow 0$ we obtain the right hand side of (6.2). A similar argument can be applied to prove the other part of (6.2).

Proof of Theorem 6.1. We shall first prove the theorem for the one dimensional case $n=1$. Let $w(x, t)=\int_{-\infty}^{x} G(y, t+1) d y=G(\cdot, t+1) * H$ (where $H$ is the Heaviside function and $*$ denotes convolution), so that

$$
w_{t}-w_{x x}=\left(G_{t}(\cdot, t+1)-G_{x x}(\cdot, t+1)\right) * H=0 \quad \text { for } t \geq 0 .
$$

The function $z^{-}(x, t) \equiv w(x+t, t)$ satisfies $z_{t}^{-}=z_{x x}^{-}+z_{x}^{-}$. Since $z^{-}$is monotone increasing in $x$ for fixed $t, z_{x}^{-}=\left|z_{x}\right|$,

$$
z_{t}^{-}=z_{x x}^{-}+\left|z_{x}^{-}\right| \text {. }
$$


Similarly, $z^{+}(x, t) \equiv w(-(x-t), t)$ satisfies $z_{t}^{+}=z_{x x}^{+}-z_{x}^{+}$, and since $z^{+}$is monotone decreasing in $x$ for fixed $t, z_{x}^{+}=-\left|z_{x}^{+}\right|$so,

$$
z_{t}^{+}=z_{x x}^{+}+\left|z_{x}^{+}\right| \text {. }
$$

We note that

$$
\begin{aligned}
& z^{ \pm}(x, t) \rightarrow 0 \quad \text { as } \quad x \rightarrow \pm \infty \quad \text { at exponential rate, } \\
& z^{ \pm}(x, t) \rightarrow 1 \quad \text { as } \quad x \rightarrow \mp \infty \quad \text { at exponential rate. }
\end{aligned}
$$

For $a>0$, let $v^{a}(x, t)=z^{-}(x+a, t)+z^{+}(x-a, t)-1$, then $0<v^{a}(x, t)<1$, and $v^{a}$ decays to zero as $x \rightarrow \pm \infty$ uniformly on bounded time intervals. Now

$$
\begin{aligned}
& v^{a}(x, t)_{t}-v^{a}(x, t)_{x x}=z^{-}(x+a, t)_{t}-z^{-}(x+a, t)_{x x}+z^{+}(x-a, t)_{t}-z^{+}(x-a, t)_{x x} \\
& =\left|z^{-}(x+a, t)_{x}\right|+\left|z^{+}(x-a, t)_{x}\right|=\left|v^{a}(x, t)_{x}\right| \\
& \quad+2 \min \left\{\left|z^{-}(x+a, t)_{x}\right|,\left|z^{+}(x-a, t)_{x}\right|\right\} .
\end{aligned}
$$

Let $f^{a}(x, t)=2 \min \left\{\left|z^{-}(x+a, t)_{x}\right|,\left|z^{+}(x-a, t)_{x}\right|\right\}$. We claim that

$$
0<f^{a}(x, t)<\alpha^{a}(t)
$$

where $\int_{0}^{\infty} \alpha^{a}(s) d s \rightarrow 0$ as $a \rightarrow \infty$.

Indeed,

so,

$$
w_{x}(x, t)=G(x, t+1)=\frac{1}{\sqrt{4 \pi(t+1)}} e^{-\frac{x^{2}}{4(t+1)}}
$$

$$
z_{x}^{-}(x+a, t)=w_{x}(x+a+t, t)=\frac{1}{\sqrt{4 \pi(t+1)}} e^{-\frac{1}{4}\left(\frac{x+a+t}{\sqrt{t+1}}\right)^{2}} .
$$

For $x \geq 0, a>1, \frac{x+a+t}{\sqrt{t+1}} \geq \frac{a+t}{\sqrt{t+1}} \geq \max \left(\sqrt{a}, \frac{1}{2} \sqrt{t}\right)$, thus for $x \geq 0, t>0, a>1$,

$$
0<z_{x}^{-}(x, t)<e^{-\frac{1}{4} \max \left(a, \frac{t}{4}\right)} .
$$

Similarly, for $x<0, t>0, a>1$,

$$
-e^{-\frac{1}{4} \max \left(a, \frac{t}{4}\right)}<z_{x}^{+}(x, t)<0 .
$$

Thus, if we define $\alpha^{a}(t)=e^{-\frac{1}{4} \max \left(a, \frac{t}{4}\right)}$ we obtain

$$
\int_{0}^{\infty} \alpha^{a}(s) d s=\int_{0}^{a / 4} e^{-\frac{1}{4} a} d s+\int_{a / 4}^{\infty} e^{-\frac{s}{16}} d s=\frac{a}{4} e^{-\frac{a}{4}}+16 e^{-\frac{a}{64}} \rightarrow 0 \quad \text { as } \quad a \rightarrow \infty,
$$

proving (6.3).

Given $1>\varepsilon>0$ choose $a>0$ sufficiently large so that $\int_{0}^{\infty} \alpha^{a}(s) d s<\varepsilon$. The function $u_{0}^{a}(x)=v^{a}(x, 0)$ is smooth and decays exponentially as $|x| \rightarrow \infty$. From Theorems 1.1 and 2.1 we know that the solution $u^{a}(x, t)$ of $u_{t}^{a}=u_{x x}^{a}+\left|\nabla u^{a}\right|, u^{a}(x, 0)=$ $u_{0}^{a}(x)$ is Lipschitz continuous in the strip $\mathbb{R} \times[0, T]$ and $\left\|u^{a}(\cdot, t)\right\|_{1}$ is uniformly bounded on each interval $[0, T]$. Furthermore, $u^{a}(x, t) \rightarrow 0$ as $|x| \rightarrow \infty$ uniformly in such time intervals. Thus, comparing $u^{a}$ to $v^{a}$ by Lemma 6.2 , one obtains

$$
\left|v^{a}(x, t)-u^{a}(x, t)\right|<\varepsilon .
$$

Let $R(\varepsilon, t)$ be such that $v^{a}(x, t)>1-\varepsilon$ for $|x| \leq R(\varepsilon, t)$. 
It is clear from the definition of $v^{a}$ that $R(\varepsilon, t)$ grows like $t-\sqrt{t}$, thus $u^{a}(x, t)>$ $1-2 \varepsilon$ on a set of size proportional to $t-\sqrt{t}$, hence $\left\|u^{a}(\cdot, t)\right\|_{1} \geq c \cdot t$, which completes the proof for the one dimensional case.

For radially symmetric functions $u=u(|x|, t)$, Eq. (6.1) reads,

$$
u_{t}=u_{r r}+\frac{n-1}{r} u_{r}+\left|u_{r}\right| \text {. }
$$

Let

$$
P^{a}(r, t)= \begin{cases}z^{+}(2-a, t), & 0 \leq r \leq 2 \\ z^{+}(r-a, t), & r>2\end{cases}
$$

and denote $\mathcal{L} u=u_{r r}+\left|u_{r}\right|$.

Then for $r<2, \quad \mathcal{L} P^{a}=0$ so,

$$
P_{t}^{a}(r, t)-\mathcal{L} P^{a}(r, t)=P_{t}^{a}(2, t) .
$$

For $r>2$,

$$
P_{t}^{a}(r, t)-\mathcal{L} P^{a}(r, t)=-\frac{n-1}{r} P_{r}^{a}(r, t)
$$

Let $\delta(x)$ be a non-negative $C_{0}^{\infty}([-1,1])$ function satisfying $\int_{-\infty}^{\infty} \delta(x) d x=1$, and let

$$
v^{a}(\cdot, t)=P^{a}(\cdot, t) * \delta(\cdot) .
$$

For $r \leq 1$ we have $v^{a}(r, t)=P^{a}(r, t)$, hence,

$$
v_{t}^{a}-\mathcal{L} v^{a}=P_{t}^{a}(2, t),
$$

and for $r \geq 1$,

$$
v_{t}^{a}-\mathcal{L} v^{a}=-\frac{n-1}{r} P_{r}^{a} * \delta
$$

so

$$
v_{t}^{a}-\mathcal{L} v^{a}=g(r, t)
$$

where

$$
|g(r, t)| \leq c \cdot \max _{r \geq 1}\left\{P_{t}^{a}, P_{r}^{a}\right\}, \quad c=c\left(n,\|\delta(r)\|_{\infty}\right)
$$

As before, we would like to find an integrable bound $\alpha(t)$ for the right hand side of (6.8), and show that $\int_{0}^{\infty} \alpha(s) d s$ can be made arbitrarily small if $a$ is chosen sufficiently large. 
Now, in terms of the one-dimensional heat kernel $G$,

$$
\begin{aligned}
P_{t}^{a}(r, t) & =\frac{\partial}{\partial t} z^{+}(r-a, t)=\frac{\partial}{\partial t} \int_{-(r-a-t)}^{\infty} G(y, t+1) d y \\
& =\frac{\partial}{\partial t} \int_{a-r+t}^{\infty} \frac{1}{\sqrt{t+1}} G\left(\frac{y}{\sqrt{t+1}}, 1\right) d y=\frac{\partial}{\partial t} \int_{\frac{a-r+t}{\sqrt{t+1}}}^{\infty} G(z, 1) d z \\
& =-G\left(\frac{a-r+t}{\sqrt{t+1}}, 1\right) \frac{\partial}{\partial t}\left(\frac{a-r+t}{\sqrt{t+1}}\right) \\
& =-\frac{1}{\sqrt{4 \pi}} e^{-\frac{(a-r-t)^{2}}{4(t+1)}}\left[-\frac{1}{2} \frac{a-r}{(t+1)^{3 / 2}}+\frac{1}{2} \frac{1}{(t+1)^{1 / 2}}\right],
\end{aligned}
$$

so,

$$
\left|P_{t}^{a}(r, t)\right| \leq C \cdot e^{-\frac{a-(r+1)}{4}} e^{-\frac{t+1}{4}}
$$

Similarly,

$$
P_{r}^{a}(r, t)=\frac{\partial}{\partial r} \int_{\frac{a-r+t}{\sqrt{t+1}}}^{\infty} G(z, 1) d z=-G\left(\frac{a-r+t}{\sqrt{t+1}}, 1\right) \cdot\left(-\frac{1}{\sqrt{t+1}}\right) .
$$

It is not hard to see that

$$
\frac{1}{r} G\left(\frac{a-r+t}{\sqrt{t+1}}, 1\right) \text { is a decreasing function of } r,
$$

so that for $r \geq 1$,

$$
\left|\frac{n-1}{r} P_{r}^{a}(r, t)\right| \leq c \cdot \frac{1}{\sqrt{t+1}} e^{-\frac{a-3}{4}} e^{-\frac{t+1}{4}} .
$$

It follows from (6.8) that the right hand side of (6.7) can be bounded by

$$
\alpha(t)=c \cdot e^{-\frac{a}{4}} \cdot e^{-\frac{t}{4}} .
$$

Let $u^{a}(x, t)$ be the solution to (6.1) with

$$
u^{a}(x, 0)=v^{a}(|x|, 0) .
$$

An application of Lemma 6.2 to $u^{a}(x, t), v^{a}(|x|, t)$ yields the required result in the same way as in the one dimensional case.

\section{Periodic Initial Conditions}

Let $T^{n}$ denote the $n$-dimensional torus $\mathbb{R}^{n} / \mathbb{Z}^{n}$. We would like to study the Cauchy problem (1.1) on $T^{n}$.

It is evident from the fact that

$$
G(x, t)=\frac{1}{(4 \pi t)^{n / 2}} e^{-\frac{x^{2}}{4 t}}
$$

is the heat kernel in $\mathbb{R}^{n}$, that

$$
H(x, t)=\frac{1}{(4 \pi t)^{n / 2}} \cdot \sum_{m \in \mathbb{Z}^{n}} e^{-\frac{(x+m)^{2}}{4 t}}
$$

is the heat kernel on $T^{n}$. 
It is not hard to verify that Theorem 1 in [B], as well as Theorems 1.1, 3.1, 4.1 and Corollary 4.5 of this paper, carry over to this case. This is achieved by replacing the heat kernel $G$ and integrations over $\mathbb{R}^{n}$ by the heat kernel $H$ and integrations over $T^{n}$. Thus the following theorem is obtained.

Theorem 7.1. For $u_{0} \in L^{1}\left(T^{n}\right)$ the Cauchy problem (1.1) on $T^{n}$ has a unique solution $u$ on $T^{n} \times \mathbb{R}_{+}$, having the following properties.

$$
\begin{gathered}
u(\cdot, t) \in C\left(\overline{\mathbb{R}}_{+}, L^{1}\left(T^{n}\right)\right), u(x, 0)=u_{0}(x) \text { a.e. } x \in T^{n}, \\
u(\cdot, t) \in L_{\text {loc }}^{1}\left(\overline{\mathbb{R}}_{+}, W^{1,1}\left(T^{n}\right) \cap L_{\mathrm{loc}}^{\infty}\left(\mathbb{R}_{+}, W^{1,1}\left(T^{n}\right)\right) .\right.
\end{gathered}
$$

Furthermore, this unique solution satisfies,

$$
u \in C^{2+\alpha, 1+\alpha}\left(T^{n} \times(\varepsilon, \infty)\right) \text { for any } \varepsilon>0,0<\alpha<1 .
$$

In particular, $u$ is a classical solution to (1.1) for $t>0$.

It follows from the strict maximum principle $[\mathrm{PW}]$ and the compactness of $T^{n}$, that if $t_{2}>t_{1}>0$,

$$
\min _{x \in T^{n}} u\left(x, t_{1}\right)<\min _{x \in T^{n}} u\left(x, t_{2}\right)<\max _{x \in T^{n}} u\left(x, t_{1}\right)
$$

if $u_{0}$ is not equal to a constant a.e.

Theorem 7.2. Let $u_{0} \in L^{1}\left(T^{n}\right)$ and let $u(x, t)$ be the unique solution to the Cauchy problem (1.1). Then there exists a constant ess $\inf u_{0} \leq c\left(u_{0}, \mu\right) \leq e s s \sup u_{0}$ such that

$$
\left\|u(\cdot, t)-c\left(u_{0}, \mu\right)\right\|_{L^{\infty}\left(T^{n}\right)} \rightarrow 0 \quad \text { as } \quad t \rightarrow \infty .
$$

Before we prove the theorem we recall the following theorem (see [GT], Theorem 7.17).

Theorem. Let $u \in W_{0}^{1, p}(\Omega), p>n$. Then $u \in C^{\gamma}(\bar{\Omega})$, where $\gamma=1-n / p$. Furthermore, for any ball with radius $R, B_{R}$

$$
\underset{\Omega \cap B_{R}}{O S C u} \leq c \cdot R^{\gamma}\|\nabla u\|_{p}
$$

where $c=c(n, p)$.

Proof of Theorem 7.2. Fix $\varepsilon>0$. Corollary 4.5 gives, for $t \geq \varepsilon$,

$$
\max _{x \in T^{n}}|\nabla u(x, t)| \leq \max _{x \in T^{n}}|\nabla u(x, \varepsilon)| \equiv \alpha .
$$

Now,

$$
\|\nabla u(\cdot, t)\|_{p} \leq\left(\max _{x \in T^{n}}|\nabla u(x, t)|\right)^{1-1 / p}\|\nabla u(\cdot, t)\|_{1}^{1 / p}
$$

so by (7.5) and (7.6), for $p>n$,

$$
\underset{T^{n}}{O S C u}(\cdot, t) \leq c \alpha^{\frac{p-1}{p}}\|\nabla u(\cdot, t)\|_{1}^{1 / p} .
$$

Let

$$
\bar{u}_{\mu}(t)=\max _{x \in T^{n}} u(x, t) \quad \text { and } \quad \underline{u}_{\mu}(t)=\min _{x \in T^{n}} u(x, t) .
$$

We have by (7.7),

$$
\|\nabla u(\cdot, t)\|_{1} \geq\left\{\left[\bar{u}_{\mu}(t)-\underline{u}_{\mu}(t)\right] / c \cdot \alpha^{\frac{p-1}{p}}\right\}^{p} .
$$


Changing $u$ to $-u$ in (1.1) is equivalent to changing $\mu$ to $-\mu$, so we can assume, without loss of generality, that $\mu<0$.

Integrate (1.1) on $T^{n}$ to obtain

$$
\begin{aligned}
\frac{\partial}{\partial t} \int_{T^{n}} u(x, t) d x & =\int_{T^{n}} \Delta u(x, t) d x+\mu \int_{T^{n}}|\nabla u(x, t)| d x=\mu \int_{T^{n}}|\nabla u(x, t)| d x \\
& \leq \mu\left\{\left[\bar{u}_{\mu}(t)-\underline{u}_{\mu}(t)\right] / c \cdot \alpha^{\frac{p-1}{p}}\right\}^{p}
\end{aligned}
$$

where the last inequality follows from (7.8) and $\mu<0$. Integrating this inequality on the interval $[\varepsilon, t]$ we have

$$
\int_{T^{n}} u(x, t) d x-\int_{T^{n}} u(x, \varepsilon) d x \leq \mu(t-\varepsilon) \cdot\left\{\left[\bar{u}_{\mu}(t)-\underline{u}_{\mu}(t)\right] / c \cdot \alpha^{\frac{p-1}{p}}\right\}^{p}
$$

since $\bar{u}_{\mu}(t)-\underline{u}_{\mu}(t)$ is monotonically decreasing (by (7.4)) and $\mu<0$.

It follows that

$$
\left\{\int_{T^{n}} u(x, t) d x-\int_{T^{n}} u(x, \varepsilon) d x\right\} \cdot c^{p} \alpha^{p-1} / \mu(t-\varepsilon) \geq\left[\bar{u}_{\mu}(t)-\underline{u}_{\mu}(t)\right]^{p} .
$$

Thus, since $u$ is bounded and $T^{n}$ has finite volume, there exists a constant $c$ s.t. $\bar{u}_{\mu}(t)-\underline{u}_{\mu}(t) \leq c / t^{1 / p}$, hence $u(x, t)$ tends uniformly to a constant which lies (by (7.4)) between $\underline{u}_{\mu}(\varepsilon)$ and $\bar{u}_{\mu}(\varepsilon)$.

To conclude the proof we have to show that

$$
\operatorname{ess} \inf u_{0} \leq \underline{u}_{\mu}(\varepsilon) \leq \bar{u}_{\mu}(\varepsilon) \leq \operatorname{ess} \sup u_{0}
$$

Let $u_{0}^{\eta}=u_{0} * \delta_{\eta}$ where $\delta_{\eta}$ is the standard mollifier. Clearly,

$$
\text { ess inf } u_{0} \leq \min u_{0}^{\eta} \leq \max u_{0}^{\eta} \leq \operatorname{ess} \sup u_{0}
$$

Let $u^{\eta}$ be the classical solution to (1.1) with initial condition $u^{\eta}(x, 0)=u_{0}^{\eta}(x)$. Then, by the maximum principle

$$
\min u_{0}^{\eta} \leq u^{\eta}(x, \varepsilon) \leq \max u_{0}^{\eta} .
$$

Since, in view of Theorem 2.1, $u^{\eta}(x, \varepsilon) \rightarrow u(x, \varepsilon)$ in $L^{1}\left(T^{n}\right)$ as $\eta \rightarrow 0$,

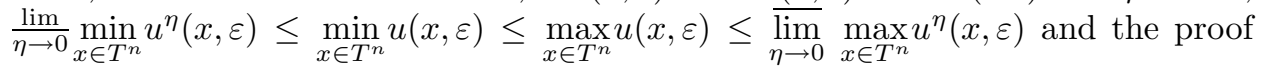
is complete.

Theorem 7.3. Let $u_{0} \in L^{1}\left(T^{n}\right)$ and let $u_{\mu}(x, t)$ be the unique solution to (1.1) with parameter $\mu$. Then,

$$
\lim _{\mu \rightarrow-\infty} u_{\mu}(x, t)=e s s \inf u_{0}, \quad \lim _{\mu \rightarrow \infty} u_{\mu}(x, t)=e s s \sup u_{0} .
$$

In particular,

$$
\lim _{\mu \rightarrow-\infty} c\left(u_{0}, \mu\right)=e s s \inf u_{0}, \quad \lim _{\mu \rightarrow \infty} c\left(u_{0} \mu\right)=e s s \sup u_{0}
$$

(where $c\left(u_{0}, \mu\right)$ is as in Th. 7.2).

In order to prove the theorem we need the following lemma, concerning the monotonicity property of (1.1). 
Lemma 7.4. Let $u_{0}, v_{0} \in C^{2}\left(T^{n}\right), \quad \mu_{1} \geq \mu_{2}$. Let $u, v$ be the bounded classical solutions of

$$
\begin{gathered}
u_{t}=\Delta u+\mu_{1}|\nabla u|, \quad u(x, 0)=u_{0}(x), \\
v_{t}=\Delta v+\mu_{2}|\nabla v|, \quad v(x, 0)=v_{0}(x) .
\end{gathered}
$$

$$
\text { If } u_{0}(x) \geq v_{0}(x), x \in T^{n} \text {, then } u(x, t) \geq v(x, t) \text { for all }(x, t) \in T^{n} \times \mathbb{R}_{+} \text {. }
$$

Proof. The function $w=u-v+\varepsilon t, \varepsilon>0$, satisfies the equation

$$
w_{t}=\Delta w+\mu_{2}(|\nabla u|-|\nabla v|)+\left(\mu_{1}-\mu_{2}\right)|\nabla u|+\varepsilon .
$$

We claim that $w$ cannot attain a minimum for $t>0$, since at such a point $(x, t), \quad w_{t} \leq 0, \quad \Delta w \geq 0, \quad \nabla w=\nabla u-\nabla v=0$, contradicting (7.14). Thus $(u-v)(x, t)+\varepsilon t \geq \min \left(u_{0}-v_{0}\right) \geq 0$ for any $\varepsilon>0$.

Corollary 7.5. The above comparison principle is valid also under the assumption $u_{0}, v_{0} \in L^{1}\left(T^{n}\right)$.

Proof. If $u_{0}(x) \geq v_{0}(x)$, then there exist sequences of smooth functions $u_{0}^{m}(x) \leq$ $v_{0}^{m}(x)$ s.t. $u_{0}^{m}(x) \rightarrow u_{0}(x)$ and $v_{0}^{m}(x) \rightarrow v_{0}(x)$ in $L^{1}\left(T^{n}\right)$ as $m \rightarrow \infty$.

Let $u^{m}(x, t), v^{m}(x, t)$ be the solutions to (7.12), (7.13), respectively. They satisfy $u^{m}(x, t) \geq v^{m}(x, t)$, by the lemma, and satisfy $u^{m}(\cdot, t) \rightarrow u(\cdot, t), v^{m}(\cdot, t) \rightarrow v(\cdot, t)$ in $L^{1}\left(T^{n}\right)$ as $n \rightarrow \infty$ by Theorem 2.1. Since $u(x, t), v(x, t)$ are continuous for $t>0$ (Th. 4.1) it follows that $u(x, t) \geq v(x, t)$, as required.

Proof of Theorem 7.3. We will prove the theorem for $\mu \rightarrow-\infty$ (the proof for $\mu \rightarrow \infty$ is obtained by changing $u$ to $-u$ ). Let $\mu<-1$, so that by Corollary $7.5 u_{\mu}(x, \varepsilon) \leq u_{-1}(x, \varepsilon)$ for $\varepsilon>0$. Let $\tilde{u}_{\mu}(x, t)$ be a solution to (1.1) with initial condition $u_{-1}(x, \varepsilon)$ at $t=\varepsilon$. Thus the above lemma gives $\tilde{u}_{\mu}(x, t) \geq u_{\mu}(x, t), t \geq \varepsilon$. Let $\alpha=\left\|\nabla u_{-1}(\cdot, \varepsilon)\right\|_{L^{\infty}\left(T^{n}\right)}<\infty, \beta=\left\|u_{-1}(\cdot, \varepsilon)\right\|_{L^{\infty}\left(T^{n}\right)}<\infty$. Corollary 4.5 gives

$$
\left\|\nabla \tilde{u}_{\mu}\right\|_{L^{\infty}\left(T^{n} \times[\varepsilon, \infty)\right)} \leq n \alpha, \quad\left\|\tilde{u}_{\mu}\right\|_{L^{\infty}\left(T^{n} \times[\varepsilon, \infty)\right)} \leq \beta,
$$

for all $\mu \leq-1$. In view of $(7.10)$,

$$
\overline{\tilde{u}}_{\mu}(t)-\underline{\tilde{u}}_{\mu}(t) \leq(2 \beta)^{1 / p} \cdot c \cdot \alpha^{\frac{p-1}{p}} \cdot[|\mu|(t-\varepsilon)]^{-1 / p},
$$

and from Corollary 7.5,

$$
\begin{aligned}
\overline{\tilde{u}}_{\mu}(t) & \leq \underline{\tilde{u}}(t)+(2 \beta)^{1 / p} \cdot c \cdot \alpha^{\frac{p-1}{p}}[|\mu|(t-\varepsilon)]^{-1 / p} \\
& \leq \underline{u}_{-1}(t)+(2 \beta)^{1 / p} \cdot c \cdot \alpha^{\frac{p-1}{p}} b \cdot[|\mu|(t-\varepsilon)]^{-1 / p} .
\end{aligned}
$$

so

$$
\bar{u}_{\mu}(2 \varepsilon) \leq \overline{\tilde{u}}_{\mu}(2 \varepsilon) \leq \underline{u}_{-1}(2 \varepsilon)+(2 \beta)^{1 / p} \cdot c \cdot \alpha^{\frac{p-1}{p}}[|\mu| \cdot \varepsilon]^{-1 / p} .
$$

Thus, $\varlimsup_{\mu \rightarrow-\infty} \bar{u}_{\mu}(2 \varepsilon) \leq \underline{u}_{-1}(2 \varepsilon)$. Now (7.4) and $u_{-1}(\cdot, t) \underset{t \rightarrow 0}{\rightarrow} u_{0}$ in $L^{1}$ imply $\underline{u}_{-1}(t) \underset{t \rightarrow 0}{\rightarrow} \operatorname{ess} \inf u_{0}$. On the other hand, by (7.11),

$$
\operatorname{essinf} u_{0} \leq \lim _{\mu \rightarrow-\infty} \bar{u}_{\mu}(t) \leq \inf _{\varepsilon<t} \lim _{\mu \rightarrow-\infty} \bar{u}_{\mu}(2 \varepsilon) \leq \inf _{\varepsilon<t} \underline{u}_{-1}(2 \varepsilon)=\operatorname{essinf} u_{0}
$$

and we are done. 


\section{APPENDIX}

Convergence of a Numerical Scheme. Consider the lattice $\Sigma$ consisting of the points

$$
(\underline{x}, t)=\left(j_{1} h, j_{2} h, \ldots, j_{n} h, m k\right), \quad j_{i} \in \mathbb{Z}, i=1, \ldots, n, m \in \mathbb{N},
$$

where $h, k$ are fixed positive constants. Let $\underline{j}=\left(j_{1}, \ldots, j_{n}\right)$ and let $v_{j}^{m}$ be the value of the function $v$ at $(\underline{j}, m)$. Define operators $E_{i}$ such that

$$
E_{i} v_{\underline{j}}^{m}=v_{\left(j_{1}, ., j_{i-1}, j_{i}+1, j_{i+1}, \ldots, j_{n}\right)}^{m} .
$$

We consider the numerical scheme,

$$
v_{\underline{j}}^{m+1}=v_{\underline{j}}^{m}+\frac{k}{h^{2}} \sum_{i=1}^{n}\left[E_{i}-2 I+E_{i}^{-1}\right] v_{\underline{j}}^{m}+\frac{\mu}{2} \frac{k}{h} \sqrt{\sum_{i=1}^{n}\left\{\left[E_{i}-E_{i}^{-1}\right] v_{\underline{j}}^{m}\right\}^{2}} .
$$

As initial conditions for the numerical scheme we take

$$
v_{\underline{j}}^{0}=v_{0}(\underline{j} \cdot h) .
$$

Theorem A.1. Let $v_{0} \in L^{\infty}\left(\mathbb{R}^{n}\right)$. If $\lambda \equiv \frac{h}{k^{2}}<\frac{1}{2 n}$ and $\left|\frac{1}{2} h \mu\right|<1$, then

$$
\sup _{\underline{j} \in \mathbb{Z}^{n}, m \in \mathbb{N}}\left|v_{\underline{j}}^{m}\right| \leq\left\|v_{0}\right\|_{\infty} \text {. }
$$

Proof. $v_{\underline{j}}^{m+1}=(1-2 n \lambda) v_{\underline{j}}^{m}+\lambda \sum_{i=1}^{n}\left(E_{i}+E_{i}^{-1}\right) v_{\underline{j}}^{m}+\frac{1}{2} h \lambda \mu \sqrt{\sum_{i=1}^{n}\left\{\left[E_{i}-E_{i}^{-1}\right] v_{\underline{j}}^{m}\right\}^{2}}$. Now if $\left|\frac{1}{2} h \mu\right|<1$,

$$
\begin{aligned}
& \left|\sum_{i=1}^{n}\left(E_{i}+E_{i}^{-1}\right) v_{\underline{j}}^{m}+\frac{1}{2} \mu h \sqrt{\sum_{i=1}^{n}\left\{\left[E_{i}-E_{i}^{-1}\right] v_{\underline{j}}^{m}\right\}^{2}}\right| \\
& \quad \leq \sum_{i=1}^{n}\left\{\left|\left(E_{i}+E_{i}^{-1}\right) v_{\underline{j}}^{m}\right|+\frac{1}{2} h \mu\left|\left(E_{i}-E_{i}^{-1}\right) v_{\underline{j}}^{m}\right|\right\} \\
& \quad \leq 2 \sum_{i=1}^{n} \max \left\{\left|E_{i} v_{\underline{j}}^{m}\right|,\left|E_{i}^{-1} v_{\underline{j}}^{m}\right|\right\} \leq 2 n \sup \left|v_{\underline{j}}^{m}\right|,
\end{aligned}
$$

since $|a+b|+|a-b|=2 \max \{|a|,|b|\}$. Denote $\left\|v^{m}\right\|=\sup _{\underline{j} \in \mathbb{Z}^{n}}\left|v_{\underline{j}}^{m}\right|$. If $2 n \lambda<1$, then $\left\|v^{m+1}\right\| \leq(1-2 n \lambda) \quad\left\|v^{m}\right\|+2 n \lambda \quad\left\|v^{m}\right\|=\left\|v^{m}\right\|$.

Theorem A.2. Let $u(x, t)$ be a classical solution to (1.1), with $u, u_{x}, u_{x x}, u_{t}$ uniformly bounded and uniformly continuous. Fix $\lambda<\frac{1}{2 n}$, then as $h \searrow 0$ the approximate solutions $v_{\underline{j}}^{m}$ tend to $u$ in $L^{\infty}\left(\mathbb{R}^{n} \times[0, T]\right)$.

Proof. It is clear from the assumptions on $u$, and the fact that (A.1) is a consistent numerical scheme for (1.1), that for any $\varepsilon>0$, if $h>0$ is sufficiently small,

$$
\begin{aligned}
u_{\underline{j}}^{m+1}=(1 & -2 n \lambda) u_{\underline{j}}^{m}+\lambda \sum_{i=1}^{n}\left[E_{i}+E_{i}^{-1}\right] u_{\underline{j}}^{m} \\
& +\frac{1}{2} h \lambda \mu \sqrt{\sum_{i=1}^{n}\left\{\left[E_{i}-E_{i}^{-1}\right] u_{\underline{j}}^{m}\right\}^{2}}+e_{\underline{j}}^{m} \cdot k
\end{aligned}
$$

where $\left|e_{\underline{j}}^{m}\right|<\varepsilon$. Thus, 


$$
\begin{aligned}
& {\underline{u_{\underline{j}}^{m+1}}}^{m-v_{\underline{j}}^{m+1}}=(1-2 n \lambda)\left(u_{\underline{j}}^{m}-v_{\underline{j}}^{m}\right)+\lambda \sum_{i=1}^{n}\left[E_{i}+E_{i}^{-1}\right]\left[u_{\underline{j}}^{m}-v_{\underline{j}}^{m}\right] \\
& \quad+\frac{1}{2} h \lambda \mu\left[\sqrt{\sum_{i=1}^{n}\left\{\left[E_{i}-E_{i}^{-1}\right] u_{\underline{j}}^{m}\right\}^{2}}-\sqrt{\sum_{i=1}^{n}\left\{\left[E_{i}-E_{i}^{-1}\right] v_{\underline{j}}^{m}\right\}^{2}}\right]+e_{\underline{j}}^{m} \cdot k .
\end{aligned}
$$

So if $h$ is small enough by the triangle inequality for absolute values and the norm in $\mathbb{R}^{n}$, we get

$$
\begin{aligned}
\mid u_{\underline{j}}^{m+1} & -v_{\underline{j}}^{m+1}|\leq(1-2 n \lambda)| u_{\underline{j}}^{m}-v_{\underline{j}}^{m}\left|+\lambda \sum_{i=1}^{n}\right|\left[E_{i}+E_{i}^{-1}\right]\left[u_{\underline{j}}^{m}-v_{\underline{j}}^{m}\right] \mid \\
& +\frac{1}{2}|h \lambda \mu| \cdot \sum_{i=1}^{n}\left|\left[E_{i}-E_{i}^{-1}\right]\left[u_{\underline{j}}^{m}-v_{\underline{j}}^{m}\right]\right|+\left|e_{\underline{j}}^{m}\right| \cdot k \\
\leq & \left.(1-2 n \lambda) \mid u_{\underline{j}}^{m}-v_{\underline{j}}^{m}\right)+2 \lambda \sum_{i=1}^{n} \max \left\{E_{i}\left[u_{\underline{j}}^{m}-v_{\underline{j}}^{m}\right], E_{i}^{-1}\left[u_{\underline{j}}^{m}-v_{\underline{j}}^{m}\right]\right\}+\left|e_{\underline{j}}^{m}\right| \cdot k \\
\leq & \left\|u^{m}-v^{m}\right\|+\left|e_{\underline{j}}^{m}\right| \cdot k
\end{aligned}
$$

from which we get $\left\|u^{m}-v^{m}\right\| \leq\left\|u^{0}-v^{0}\right\|+\varepsilon T=\varepsilon T$. Now as $h \searrow 0$ we can take $\varepsilon \searrow 0$ and convergence is established.

\section{REFERENCES}

[AF] L. Alfonsi and F.B. Weissler, Blow-up in $\mathbb{R}^{n}$ for a parabolic equation with a damping nonlinear gradient term, in "Progress in Nonlinear Differential Equations", N.G. Lloyd et al., (Eds.), Birkhauser, 1992. MR 93f:35118

[B] M. Ben-Artzi, Global Existence and Decay for a Nonlinear Parabolic Equation, Nonlinear Analysis TMA 19 (1992), 763-768. MR 93i:35066

[BRV] S. Benachour, B. Roynette and P. Vallois, Asymptotic Estimates of Solutions of $u_{t}-$ $\frac{1}{2} \Delta u=-|\nabla u|$ in $\mathbb{R}_{+} \times \mathbb{R}^{d}, d \geq 2$, J. Funct. Anal. 144 (1997), 301-324. MR 97m:35118

[F] A. Friedman, "Partial Differential Equations of Parabolic Type", Prentice-Hall, 1964. MR 31:6062

[GT] D. Gilbarg and N.S. Trudinger, "Elliptic Partial Differential Equations of Second Order", Springer-Verlag, 1977. MR 57:13109

[PW] M. Protter and H. Weinberger, "Maximum Principles in Differential Equations", PrenticeHall, 1967. MR 36:2935

[Sh] E. Shamir, private communication.

[So] P. Souplet, Resultats d' explosion en temps fini pour une equation de la chaleur non lineaire, C.R.A.S. Paris, 321 Serie I, (1995), 721-726. MR 96h:35093

[SW] P. Souplet and F.B. Weissler, Poincaré's inequality and global solutions of a nonlinear parabolic equation, Annales Inst. H. Poincaré - Anal. Nonlin. 16 (1999), 337-373.

Institute of Mathematics, Hebrew University, Jerusalem 91904, Israel

E-mail address: mbartzi@math.huji.ac.il

Courant Institute of Mathematical Sciences, New York, New York 10012

E-mail address: goodman@cims.nyu.ed

Courant Institute of Mathematical Sciences, New York, New York 10012 